\title{
Preliminary Findings from an Investigation of Zika Virus Infection in a Patient with No Known Risk Factors — Utah, 2016
}

\author{
Carolyn Brent ${ }^{1,2}$; Angela Dunn, $\mathrm{MD}^{3}$; Harry Savage, $\mathrm{PhD}^{4}$; Ary Faraji, PhD 5 ; Mike Rubin, $\mathrm{MD}^{6}$; Ilene Risk, MPA ${ }^{1}$; Wendy Garcia ${ }^{7}$; \\ Margaret Cortese, $\mathrm{MD}^{8}$; Shannon Novosad, MD ; Elisabeth Raquel Krow-Lucal, PhD ${ }^{4}$; Jacqueline Crain ${ }^{2,3}$; Mary Hill, MPH${ }^{1}$; Annette Atkinson, MS ${ }^{10}$; \\ Dallin Peterson $^{3}$; Kimberly Christensen ${ }^{10}$; Melissa Dimond, $\mathrm{MPH}^{3}$; J. Erin Staples, MD ${ }^{4}$; Allyn Nakashima, $\mathrm{MD}^{3}$
}

On September 13, 2016, this report was posted as an MMWR Early Release on the MMWR website (http://www.cdc.gov/mmwr).

On July 12, 2016, the Utah Department of Health (UDOH) was notified by a clinician caring for an adult (patient A) who was evaluated for fever, rash, and conjunctivitis that began on July 1. Patient A had not traveled to an area with ongoing Zika virus transmission; had not had sexual contact with a person who recently traveled; and had not received a blood transfusion, organ transplant, or mosquito bites (1). Patient A provided care over several days to an elderly male family contact (the index patient) who contracted Zika virus abroad. The index patient developed septic shock with multiple organ failure and died in the hospital on June 25, 2016. The index patient's blood specimen obtained 2 days before his death had a level of viremia approximately 100,000 times higher than the average level reported in persons infected with Zika virus (2). Zika virus infection was diagnosed in patient $A$ by real-time reverse transcription-polymerase chain reaction (rRT-PCR) testing on a urine specimen collected 7 days after symptom onset. In addition, a serum specimen collected 11 days after symptom onset, after patient A's symptoms had resolved, was positive for antibodies to Zika virus by Zika immunoglobulin M (IgM) capture enzyme-linked immunosorbent assay (MAC-ELISA) and had neutralizing antibodies detected by plaque-reduction neutralization testing (PRNT). Working with Salt Lake and Davis County Health Departments, UDOH requested assistance from CDC with an investigation to determine patient A's exposures and determine a probable source of infection.

The investigation consisted of four components: 1) an epidemiologic evaluation of family contacts of the index patient, 2) a serosurvey of health care workers who provided direct care to the index patient before his death, 3) a community serosurvey around the locations where the index patient had resided, and 4) active vector surveillance near the residences of the index patient and patient A. For the purpose of this investigation, a family contact was defined as a person who resided in the same household as the index patient or had direct contact with his body fluids (i.e., tears, conjunctival discharge, saliva, vomitus, urine, or stool) during the period when he was most likely viremic, including a few days before his illness onset and until his death.
Nineteen family contacts, including patient A, were identified and interviewed, and provided blood or urine specimens for testing. Thirteen family contacts reported hugging and kissing the index patient's face. Five family contacts reported being present while the index patient's stool, urine, or vomitus was being cleaned. Patient A reported hugging and kissing the index patient, in a similar fashion to other family contacts, and assisted hospital personnel in holding the index patient while his stool was being cleaned, but did not have direct contact with stool. Other than patient A, all family contacts were negative for Zika virus infection by rRT-PCR or MAC-ELISA on specimens obtained roughly 2-3 weeks after last exposure.

Health care workers who provided care to the index patient and residents living within a 200-meter radius of the two homes where the index patient resided before becoming hospitalized were interviewed to assess risk factors for Zika virus infection and were offered Zika virus testing. As of August 22, 86 health care worker contacts have been identified and interviewed to assess types of patient interactions and to quantify level of exposure to the index patient's body fluids. A total of 238 households were approached, and all available and consenting household members were interviewed using a standardized questionnaire about risk factors for mosquitoborne transmission. All health care workers and community members who provided blood specimens are being tested for Zika virus IgM antibodies using a MAC-ELISA. Urine specimens were also collected from any persons who reported Zika virus-like symptoms in the 14 days before their interview. Testing is incomplete, but as of August 22 it has not revealed any persons with Zika virus infections.

Local mosquito abatement districts worked in collaboration with vector entomologists from CDC to conduct larval and adult mosquito surveillance near the index patient's and patient A's residences. Door-to-door surveys around neighborhood homes were conducted and a variety of mosquito traps (e.g., Biogents Sentinel, gravid, light traps baited with carbon dioxide, and ovitraps) were deployed. Larval specimens obtained in the field were reared to the adult stage for identification. Adult mosquitoes are in the process of being identified and tested for Zika virus RNA by rRT-PCR, but no Aedes aegypti or Aedes albopictus mosquitoes have been identified as part of this investigation. 
It remains unclear how patient $A$ was infected; however patient A was known to have had close contact (i.e. kissing and touching) with the index patient while the index patient's viral load was found to be very high. Although it is not certain that these types of close contact were the source of transmission, family contacts should be aware that blood and body fluids of severely ill patients might be infectious. Given recognition of high levels of viremia during illness, it is essential that health care workers continue to apply standard precautions while caring for all patients, including those who might have Zika virus disease (3).

\section{Acknowledgments}

Jeanmarie Mayer, MD, Sankar Swaminathan, MD, University of Utah Hospital; Dagmar Vitek, MD, Tara Brunatti, MSN-PH, Andrea Price, RN, Andrew Dibb, MPH, Salt Lake County Health Department, Utah; Bryan Burk, Utah Public Health Laboratory; Greg White, PhD, Salt Lake City Mosquito Abatement District, Utah; Ryan Lusty, Magna Mosquito Abatement District, Utah; Brian Hougaard, Eric Gardner, MSc, South Salt Lake Valley Mosquito Abatement District, Utah; Gary Hatch, Mosquito Abatement District Davis, Utah; Bryan Christensen, PhD; Scott Fridkin, MD, Marvin Godsey, Jr., MS, Susan Hills, MBBS, Nina Johnson, Amy
Lambert, PhD, Kathy Seiber, MS, Ian Williams, MD; the Emergency Operation Center's Zika Virus Response, CDC.

${ }^{1}$ Salt Lake County Health Department, Salt Lake City, Utah; ${ }^{2}$ Public Health
Associates Program Office, The Office for State, Tribal, Local and Territorial
Support, CDC; ${ }^{3}$ Utah Department of Health; ${ }^{4}$ Division of Vector-Borne
Diseases, National Center for Emerging and Zoonotic Infectious Diseases,
CDC; ${ }^{5}$ Salt Lake City Mosquito Abatement District, Salt Lake City, Utah;
${ }^{6}$ University of Utah Hospital, Salt Lake City, Utah; ${ }^{7}$ Davis County Health
Department, Clearfield, Utah; ${ }^{8}$ Division Of Viral Diseases, National Center
For Immunization And Respiratory Diseases, CDC; ${ }^{9}$ Division of Healthcare
Quality Promotion, National Center for Emerging and Zoonotic Infectious
Diseases, CDC; ${ }^{10}$ Utah Public Health Laboratory, Taylorsville, Utah.

Corresponding author: Carolyn Brent, cbrent@slco.org, 385-468-3918.

\section{References}

1. CDC. Zika virus transmission and risks. Atlanta, GA: US Department of Health and Human Services, CDC; 2016. http://www.cdc.gov/zika/ transmission/index.html

2. Lanciotti RS, Kosoy OL, Laven JJ, et al. Genetic and serologic properties of Zika virus associated with an epidemic, Yap State, Micronesia, 2007. Emerg Infect Dis 2008;14:1232-9. http://dx.doi.org/10.3201/ eid 1408.080287

3. CDC. 2007 guideline for isolation precautions: preventing transmission of infectious agents in healthcare settings. Atlanta, GA: US Department of Health and Human Services, CDC; 2007. http://www.cdc.gov/hicpa c/2007IP/2007isolationPrecautions.html 